\title{
From our readers
}

\section{DISLOCATION PROPAGATION VERSUS DISLOCATION NUCLEATION}

To the editor - In the recent News and Views article "Less is more" by E. Ma, T. D. Shen and X. L. Wu ${ }^{1}$, the authors have incorrectly interpreted our molecular dynamics simulation results. In order to support their belief that the propagation of dislocations is easier than the nucleation of dislocations, the authors refer to our recent molecular dynamics simulation results ${ }^{2}$, stating "In fact, their movement is easier under applied stresses than the generation of new dislocations from grain boundaries."

Our paper does not support this statement. On the contrary, our simulations and experiments ${ }^{3,4}$ suggest the importance of dislocation propagation. Reporting the details of the nucleation and propagation obtained from molecular dynamics, we demonstrated that dislocation propagation is hindered by the ledges in the grain boundaries, and that propagation can be as important as nucleation. Our paper ${ }^{2}$ ends with the sentence "The present paper demonstrates that dislocation propagation can play as important a role as dislocation nucleation and that this should be taken into account in any continuum model for the rate-controlling mechanism of dislocation activity in nanocrystalline materials".

The opinion adopted by the authors in their News and Views piece, that nucleation is the rate-limiting process, is an extremely strong statement that is still not supported by experiments. Moreover, their statement seems not to be in accordance with discussions in the authors' own experimental papers ${ }^{5,6}$.

At the current level of understanding, the rate-limiting process for plastic deformation is still an open question. Suggestions from molecular dynamics simulations such as those presented in ref. 2 should motivate further in-depth experimental research exploring the details of the deformation mechanism aiming to determine the character of the rate-limiting processes in grain-boundary-dominated structures, an input of utmost importance for developing reliable mesoscopic models.

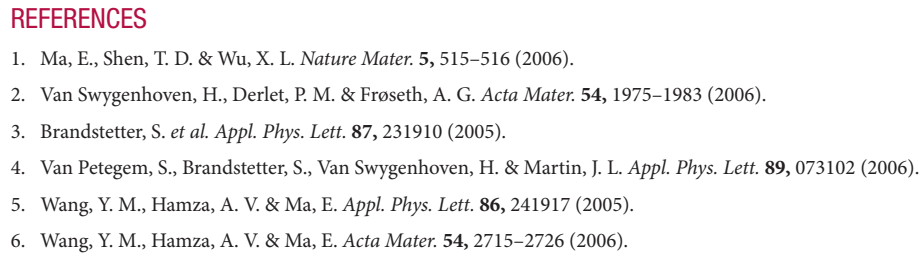

Helena Van Swygenhoven

Paul Scherrer Institute, CH-5232 Villigen PSI, Switzerland

e-mail: helena.vanswygenhoven@psi.ch

Authors' response - This issue needs to be analysed in the context from which it was quoted. In that paragraph of our text ${ }^{1}$, we discuss the dislocations accumulated during sample preparation inside the grain volume between the grain boundaries. Such is the case of ref. 2 that we were commenting on. These grains, above $100 \mathrm{~nm}$ in size, are relatively large compared with those modelled in molecular dynamics simulations, and were produced by repeated rolling. As such, there are pre-existing dislocations in the interior of the grains $\mathbf{s}^{1,2}$. Some of these stored dislocations can move relatively easily on subsequent tensile testing with little hindrance from the high-angle grain boundaries and without the need to nucleate new dislocations. The plastic strains observed in the tensile tests were also partly attributed to the propagation of these ready-to-go dislocations ${ }^{2}$. On the other hand, dislocation nucleation, which has been studied before for dislocation emission from the grain boundaries (hence the citation of the paper by Van Swygenhoven et al. ${ }^{3}$ ), requires high stresses at the grain boundaries and becomes necessary and dominant in small nanocrystalline grains with clean interiors, or after annealing wipes away those dislocations introduced into the grains during rolling ${ }^{2}$.

As for the general importance of dislocation nucleation versus mobility, our article did not hold the opinion that nucleation must always be the rate-limiting process in plastic flow. In fact, we have mentioned this in a later paragraph, and previously discussed several mechanisms ${ }^{4-6}$ for nanograins (especially those with grain size well below $100 \mathrm{~nm}$ ). We proposed that dislocation-grain-boundary interactions, including dislocation emission from the grain boundaries as well as dislocation motion overcoming hindrance in the grain-boundary regions, can become the thermally activated, rate-limiting processes $^{4-6}$.

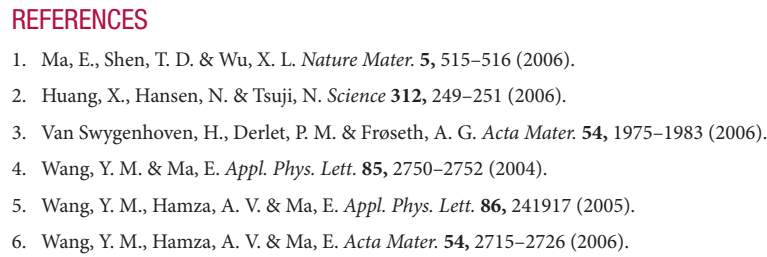

\section{E. Ma ${ }^{\mathbf{l}^{*}}$, T. D. Shen ${ }^{2}$ and X. L. Wu ${ }^{3}$}

${ }^{1}$ Department of Materials Science and Engineering, Johns Hopkins University, Baltimore, Maryland 21218, USA

${ }^{2}$ Materials Science and Technology Division, Los Alamos National Laboratory, Los Alamos, New Mexico 87545, USA

${ }^{3}$ State Key Laboratory of Nonlinear Mechanics, Institute of Mechanics, Chinese Academy of Sciences, Beijing 100080, China

*e-mail:ema@jhu.edu 


\section{From our readers}

\section{DISLOCATION PROPAGATION VERSUS DISLOCATION NUCLEATION}

To the editor - In the recent News and Views article "Less is more" by E. Ma, T. D. Shen and X. L. Wu ${ }^{1}$, the authors have incorrectly interpreted our molecular dynamics simulation results. In order to support their belief that the propagation of dislocations is easier than the nucleation of dislocations, the authors refer to our recent molecular dynamics simulation results ${ }^{2}$, stating "In fact, their movement is easier under applied stresses than the generation of new dislocations from grain boundaries."

Our paper does not support this statement. On the contrary, our simulations and experiments ${ }^{3,4}$ suggest the importance of dislocation propagation. Reporting the details of the nucleation and propagation obtained from molecular dynamics, we demonstrated that dislocation propagation is hindered by the ledges in the grain boundaries, and that propagation can be as important as nucleation. Our paper ${ }^{2}$ ends with the sentence "The present paper demonstrates that dislocation propagation can play as important a role as dislocation nucleation and that this should be taken into account in any continuum model for the rate-controlling mechanism of dislocation activity in nanocrystalline materials".

The opinion adopted by the authors in their News and Views piece, that nucleation is the rate-limiting process, is an extremely strong statement that is still not supported by experiments. Moreover, their statement seems not to be in accordance with discussions in the authors' own experimental papers ${ }^{5,6}$.

At the current level of understanding, the rate-limiting process for plastic deformation is still an open question. Suggestions from molecular dynamics simulations such as those presented in ref. 2 should motivate further in-depth experimental research exploring the details of the deformation mechanism aiming to determine the character of the rate-limiting processes in grain-boundary-dominated structures, an input of utmost importance for developing reliable mesoscopic models.

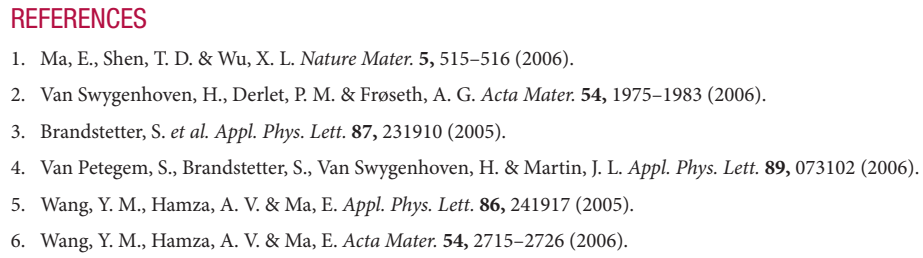

Helena Van Swygenhoven

Paul Scherrer Institute, CH-5232 Villigen PSI, Switzerland

e-mail: helena.vanswygenhoven@psi.ch

Authors' response - This issue needs to be analysed in the context from which it was quoted. In that paragraph of our text ${ }^{1}$, we discuss the dislocations accumulated during sample preparation inside the grain volume between the grain boundaries. Such is the case of ref. 2 that we were commenting on. These grains, above $100 \mathrm{~nm}$ in size, are relatively large compared with those modelled in molecular dynamics simulations, and were produced by repeated rolling. As such, there are pre-existing dislocations in the interior of the grains $\mathbf{s}^{1,2}$. Some of these stored dislocations can move relatively easily on subsequent tensile testing with little hindrance from the high-angle grain boundaries and without the need to nucleate new dislocations. The plastic strains observed in the tensile tests were also partly attributed to the propagation of these ready-to-go dislocations ${ }^{2}$. On the other hand, dislocation nucleation, which has been studied before for dislocation emission from the grain boundaries (hence the citation of the paper by Van Swygenhoven et al. ${ }^{3}$ ), requires high stresses at the grain boundaries and becomes necessary and dominant in small nanocrystalline grains with clean interiors, or after annealing wipes away those dislocations introduced into the grains during rolling ${ }^{2}$.

As for the general importance of dislocation nucleation versus mobility, our article did not hold the opinion that nucleation must always be the rate-limiting process in plastic flow. In fact, we have mentioned this in a later paragraph, and previously discussed several mechanisms ${ }^{4-6}$ for nanograins (especially those with grain size well below $100 \mathrm{~nm}$ ). We proposed that dislocation-grain-boundary interactions, including dislocation emission from the grain boundaries as well as dislocation motion overcoming hindrance in the grain-boundary regions, can become the thermally activated, rate-limiting processes $^{4-6}$.

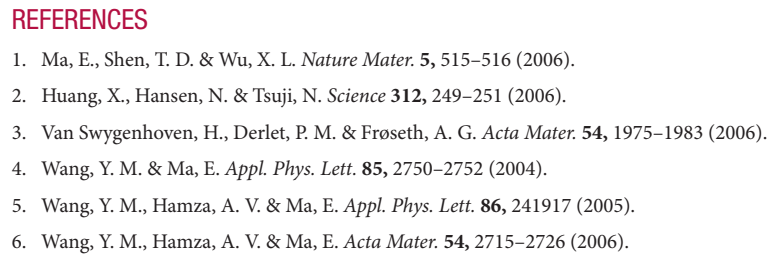

\section{E. Ma ${ }^{\mathbf{l}^{*}}$, T. D. Shen ${ }^{2}$ and X. L. Wu ${ }^{3}$}

${ }^{1}$ Department of Materials Science and Engineering, Johns Hopkins University, Baltimore, Maryland 21218, USA

${ }^{2}$ Materials Science and Technology Division, Los Alamos National Laboratory, Los Alamos, New Mexico 87545, USA

${ }^{3}$ State Key Laboratory of Nonlinear Mechanics, Institute of Mechanics, Chinese Academy of Sciences, Beijing 100080, China

*e-mail:ema@jhu.edu 